\title{
Global ocean heat transport dominated by heat export from the tropical Pacific
}

Article

Accepted Version

Forget, G. and Ferreira, D. (2019) Global ocean heat transport dominated by heat export from the tropical Pacific. Nature Geoscience, 12. pp. 351-354. ISSN 1752-0894 doi: https://doi.org/10.1038/s41561-019-0333-7 Available at https://centaur.reading.ac.uk/82528/

It is advisable to refer to the publisher's version if you intend to cite from the work. See Guidance on citing.

To link to this article DOI: http://dx.doi.org/10.1038/s41561-019-0333-7

Publisher: Nature Publishing Group

All outputs in CentAUR are protected by Intellectual Property Rights law, including copyright law. Copyright and IPR is retained by the creators or other copyright holders. Terms and conditions for use of this material are defined in the End User Agreement.

\section{www.reading.ac.uk/centaur}

\section{CentAUR}

Central Archive at the University of Reading

Reading's research outputs online 


\title{
Effective Ocean Heat Transport Revealed
}

\author{
Gaël Forget, ${ }^{1 *}$ David Ferreira, ${ }^{2}$ \\ ${ }^{1}$ Dept. of Earth, Atmospheric and Planetary Sciences, \\ Massachusetts Institute of Technology, Cambridge, MA 02139, USA. \\ ${ }^{2}$ University of Reading, Department of Meteorology, \\ PO box 243, Room 1U10, Reading, RG6 6BB, UK.
}

*To whom correspondence should be addressed; E-mail: gforget@ mit.edu.

\begin{abstract}
One of the primary mechanisms by which oceans regulate Earth's climate is through redistribution of heat. However, interpretation of ocean heat transport (OHT) has been obscured by internal heat loops that are confined within the ocean and are not directly relevant to Earth's energy budget. Here we eliminate these internal heat loops to reveal the net effects of OHT as part of the climate system, the effective OHT. Our result challenges the previous paradigm, which emphasized global conveyor belt concepts, by highlighting that poleward OHT within the Pacific is four times as large as any inter-oceanic or inter-hemispheric OHT. This shift in interpretation implies that efforts aimed at monitoring and estimating OHT changes in the Pacific should be prioritized. Furthermore, our analysis framework bridges a gap between direct estimation of OHT from oceanic measurements and indirect estimation of OHT via atmospheric energy budgets. It provides means to calibrate otherwise inconsistent OHT estimates, which will allow for more meaningful model-data comparisons.
\end{abstract}


The Ocean plays an important role in redistributing heat within the evolving climate system (1). Perhaps most importantly, it transports heat from the equator, where oceans take up heat in excess, towards higher latitudes where heat gets released to the atmosphere. This is clearly seen in observational estimates of global meridional ocean heat transport (OHT) which is directed poleward in both Hemispheres $(2,3)$. However, interpretation of measurements can be more complicated when looking at individual ocean basins since seawater can loop around land masses. This difficulty has lead to highly uncertain depictions of regional OHT contributions in the scientific literature (4), but the analysis presented here provides a framework to help reconcile previous estimates.

The two following examples illustrate how regional OHT contributions may, or may not play a role in Earth's energy budget. First, consider the case of OHT converging into an ocean basin to balance out heat release to the Atmosphere. Such OHT contributions play an important role in Earth's energy budget $(5,6)$. Second, consider a situation where seawater parcels loop around a land mass with unchanged temperatures in the ocean interior - e.g., around Australia in the counterclockwise direction. The seawater parcels would transport their internal energy northward in the Pacific only to return it southward in the Indian. Owing to internal cancellation between northward and southward OHT contributions within the ocean, such mass fluxes have no direct impact on Earth's energy budget.

Mass fluxes can thus obscure the most relevant, convergent or divergent OHT contributions. Furthermore, any oceanic section is potentially affected by this issue in ways that are not easy to predict given the complex, present-day arrangement of land masses. Freshwater may also temporarily leave the Ocean via evaporation to re-enter it elsewhere via precipitation, and closing such ocean-atmosphere loops (7) can, in and of itself, introduce non-zero mass fluxes through any oceanic section. Consequently, OHT through any oceanic section is a priori sensitive to the choice of an energy reference level (ERL; i.e., conditions of temperature, pressure, etc., 
at which energy is defined as zero by convention) which often requires careful treatment, for example to ensure energy conservation in climate models (8).

The need to adequately account for mass fluxes has long been recognized in the oceanographic literature, where various approaches have been proposed to treat non-zero mass fluxes across OHT sections $(9,10)$. One method is to adjust the net mass flux through each section to near zero $(11,12)$. Another approach is to pair sections with one another, aiming to cancel out non-zero mass flux contributions, and focus on OHT convergence between sections. This is often done by pairing various sections with Bering strait or the Indonesian Through Flow $(4,13)$. Alternatively, in cases such as Drake Passage where simply adjusting to zero mass flux is not sensible and there isn't an obvious pairing section, one may opt to introduce a distinctive temperature transport scale sometimes denoted as 'PWT' $(4,13)$. These heterogeneous treatments of OHT can render comparison amongst estimates difficult and contribute to large uncertainties and unexplained differences between estimates $(4,6)$.

Methods And Data Progress in understanding OHT can be made, instead, through a systematic decomposition of OHT over the global ocean into divergent and rotational components as done here using a gridded data product (supplementary material, SPM, sections 1-2). In practice, this simply involves computing the vertical integral and lateral divergence of the gridded OHT estimate and then solving a Poisson equation. Similar Helmholtz decomposition technics have been used in the atmospheric and oceanic literature to decompose various fluxes (14-16). Here we present a novel application of this approach to a recent observational estimate of OHT over the 1992-2011 period. This gridded OHT estimate is part of a global ocean state estimate that agrees, within expected uncertainty ranges, with the bulk of available ocean observations collected between 1992 and 2011, including millions of Argo profiles and complementary satellite data, as well as with atmospheric re-analysis estimates (17-24). 
The ocean state estimate includes global vector fields of OHT associated with dynamically consistent heat budgets, which is crucial for our purpose. This allows us, for instance, to readily verify that the divergence of OHT balances out regional air-sea heat fluxes to a good approximation while the other terms, from heat storage and the divergence of mass fluxes, are comparatively small on average over 20 years (SPM section 3). Hereafter, we denote the divergent OHT component, which approximately balances out air-sea heat fluxes, as effective OHT (effOHT). It will be compared with raw ocean heat transport (rawOHT) which also includes the rotational OHT component (rawOHT=effOHT+rotOHT). We come back to rotOHT and its interpretation later on in the paper. By convention, ERL is set to the energy of liquid water at $0^{\circ} \mathrm{C}$. Importantly, effOHT, unlike rotOHT or rawOHT, is insensitive to this choice (SPM section 4).

Transport Maps Values of effOHT and rawOHT are coarsely charted in Fig. 1 and tabulated in SPM. By construction, effOHT and rawOHT yield the exact same divergence field, shown as contours in Fig. 1. For example, 1.46 PW diverges from the tropical Pacific while $0.11 \mathrm{PW}$ converges into the Atlantic for both the red and the blue charts in Fig. 1. They are also identical when integrated all the way around the globe (e.g., black curve in Fig. 2). Nevertheless, effOHT and rawOHT provide fundamentally different perspectives on regional influences, due to the prominence of internal oceanic heat loops in rawOHT, as evidenced by Fig. 1. The rawOHT values generally fit well within the range of previously published "direct" estimates $(4,11-13$, $25)$, whereas effOHT rather corresponds to "indirect" estimates (3-6).

Owing to the presence of the Atlantic Meridional Overturning Circulation (AMOC), which brings relatively warm water into the Northern Hemisphere and returns colder water back into the Southern Hemisphere, Atlantic OHT is northward across the Equator and in both Hemispheres $(13,26,27)$ as seen in Figs. 1-2. In the Pacific, large amounts of heat enter the Ocean through the sea surface in the Tropics (black contours in Fig. 1). Based on rawOHT, most of 
this heat uptake (1.20 PW out of 1.46 PW) appears to be transferred to the Indian ocean via the Indonesian Through Flow (ITF). The Southern Ocean also stands out for its large eastward rawOHT, with values ranging between about 1 and $2 \mathrm{PW}$ seen around Antarctica. But, these values are highly sensitive to ERL choices, so much so that setting ERL to $3.5^{\circ} \mathrm{C}$, the approximate global mean ocean temperature, instead of $0^{\circ} \mathrm{C}$ suffices to reverse the direction of rawOHT over the Southern Ocean (SPM section 4). This is not the case for effOHT which is much less affected by non-zero mass fluxes (SPM sections 3-4).

Eliminating internal oceanic heat loops reduces inter-ocean exchanges to second-order terms in effOHT (red chart in Fig. 1); hence, the Pacific merely provides 0.2 PW to each of the Atlantic, Arctic, and Indian. The direction of effOHT in the South Pacific is reversed as compared with rawOHT (poleward instead of equatorward) while poleward effOHT is much smaller than rawOHT would suggest in the Indian $\left(0.23\right.$ versus $1.33 \mathrm{PW}$ at $30^{\circ} \mathrm{S}$; a factor $\approx 6$ reduction). These differences are linked to an anticlockwise loop of ocean circulation around Australia $(13,28)$ which is here estimated to contribute $\approx 1.1 \mathrm{PW}$ rawOHT with a $0^{\circ} \mathrm{C}$ ERL. Omitting this heat loop reveals that the net effect of the tropical connection between Pacific and Indian, the ITF, is only a small contribution to Earth's energy balance ( $0.10 \mathrm{PW}$ effOHT).

In the Southern Ocean, at all longitudes, effOHT is again much smaller than rawOHT, due to the omission of $\mathrm{a} \approx 1.3 \mathrm{PW}$ heat loop around Antarctica. This shift reveals that heat loss to the atmosphere in the southern Pacific sector is sustained by heat uptake in the tropical Pacific rather than by heat sources in the southern Indian sector as might have been inferred from rawOHT $(29,30)$. In fact, westward effOHT from the southern Pacific is found to sustain heat loss in the southern Indian - not the other way around. There is virtually no effOHT between the Indian and the Atlantic (0.01 PW). Finally, westward effOHT from the southern Pacific to the Atlantic via Drake Passage explains about half of the heat convergence into the South Atlantic; the other half is provided locally by heat uptake from the atmosphere. 
Meridional Transports The respective roles played by the Atlantic, Pacific, and Indian in transporting heat poleward also need to be revisited (Fig. 2). Estimates of rawOHT indeed suggest that only the Indian Ocean transports heat poleward in the Southern Hemisphere (e.g., see blue chart in Fig. 1). On the contrary, poleward effOHT in the South Pacific (0.69 PW at $\left.30^{\circ} \mathrm{S}\right)$ overpowers that in the Indian $\left(0.23 \mathrm{PW}\right.$ at $\left.30^{\circ} \mathrm{S}\right)$ as is clearly seen in Fig. 2. Furthermore, inter-hemispheric symmetry becomes evident in the Pacific based on effOHT, with poleward transport $\approx 0.7 \mathrm{PW}$ at $30^{\circ} \mathrm{N}$ and $30^{\circ} \mathrm{S}$, and near zero cross-equatorial transport $(0.02 \mathrm{PW})$.

Another noteworthy shift occurs in the Northern Hemisphere where poleward effOHT is larger in the Pacific than in the Atlantic (Fig. 2) whereas rawOHT would suggest the opposite according to our estimate (Fig. 1 and SPM). This shift reflects the omission of a $\approx 0.2 \mathrm{PW}$ heat loop which is directed northward throughout the Atlantic, crosses the Arctic, and comes back southward in the Indo-Pacific. As a result, the Pacific is found to account for two-thirds of heat converging into the Arctic (0.20 PW out of $0.29 \mathrm{PW})$ where it can participate in melting Arctic sea ice (31).

The most relevant gauge for AMOC-related OHT is provided by effOHT at the equator where wind-driven OHT is expected to be small. This yields an estimate of $0.39 \mathrm{PW}$ for the AMOC-related OHT which approximately balances out (a) $0.27 \mathrm{PW}$ atmospheric heat uptake in the southern Atlantic sector plus 0.19 PW provided by the Pacific and (b) 0.34 PW heat loss to the Atmosphere in the North Atlantic plus 0.09 PW diverging to the Arctic. Such small interhemispheric and inter-oceanic OHT magnitudes $(<0.4 \mathrm{PW})$ challenge the notion that AMOCrelated OHT must play a crucial role in regulating the global climate system (I). Poleward heat transport within the Pacific is a much bigger term ( $>1.6 \mathrm{PW}$ in total).

Subtracting 0.39 PW from effOHT throughout the Atlantic in turn yields an estimate of 0.33 PW (resp. 0.30 PW) for the northern (resp. southern) subtropical wind-driven cells, which is consistent with previous findings that the inter-hemispheric AMOC only represents $\approx 50 \%$ 
of the peak OHT in the Atlantic $(13,27)$. Interestingly, $0.30-0.33 \mathrm{PW}$ is also $\approx 50 \%$ of the corresponding value for the Pacific subtropical wind-driven cells (0.7 PW). Such proportionality is hypothesized to reflect that the Pacific is about twice as wide as the Atlantic along the Equator where oceanic heat uptake is concentrated. Hence, the gyre systems of the Pacific and Atlantic appear to redistribute tropical heat uptake with approximately the same efficiency.

\section{Heat Loops The rotational OHT component estimate (rotOHT=rawOHT-effOHT) provides a} global mapping of internal oceanic heat loops (grey contours in Fig. 3). By construction, it is divergence-free and yields zero global meridional transport. When ERL is set to $0^{\circ} \mathrm{C}$ as in Fig. 3, the patterns of rotOHT highlight familiar features of the wind-driven ocean circulation that can connect and even shape up regions of air-sea heat uptake and loss $(16,28,32)$. Subtropical gyres and the associated shallow overturning circulations notably set up the global hemispheric pattern of poleward OHT (27) and are clearly reflected in rotOHT (Fig. 3). This provides an interpretation for the westward orientation of effOHT at mid-latitudes (recall that the divergent component of a vector field is orthogonal to the scalar potential; effOHT thus goes from red to blue contours in Fig. 3) as a result of subtropical gyres releasing heat received in the tropics primarily via their western boundary currents and associated mode water formation sites (33).

Perspectives Our analysis bridges the gap between two contrasting depictions of OHT which tend to emerge either from an atmospheric perspective (3) or from an oceanic perspective (2). By deriving both of them, effOHT and rawOHT, from the same gridded data product we show that apparent conflicts amongst earlier estimates may in part reflect comparison of apples with oranges (see Fig. 1 and Tables in SPM). The Helmholtz decomposition methodology provides a framework to resolve such differences. The underlying Poisson equation approach alleviates uncertainty associated with arbitrary boundary condition and ERL choices in previous studies (see SPM section 4). Applying it to full oceanic heat budgets notably shows that inferring 
effOHT from air-sea fluxes implies an uncertainty of $\pm 0.3 \mathrm{PW}$ associated with mass fluxes and heat storage variations (SPM section 3).

A remaining challenge, however, is the lack of practical methods to accurately quantify OHT uncertainties that derive from limited ocean data coverage, uncertain air-sea fluxes, and imperfect estimation methods. Progress on this issue could be made by leveraging the Helmholtz decomposition methodology employed here to analyze OHT differences amongst ocean data assimilation products (34). From a sea-going oceanography perspective, the availability of rotOHT estimates may also help circumvent the fact that, due to the fundamentally global nature of Earth's energy budget, effective OHT cannot be observed locally by an individual section. Subtracting, for example, the rotOHT estimate shown in Fig. 3 from ship-based OHT estimates would indeed provide a systematic method to convert them into effOHT estimates - a promising alternative to the heterogeneous and sometimes ad-hoc treatments of non-zero mass fluxes found in the oceanographic literature. 


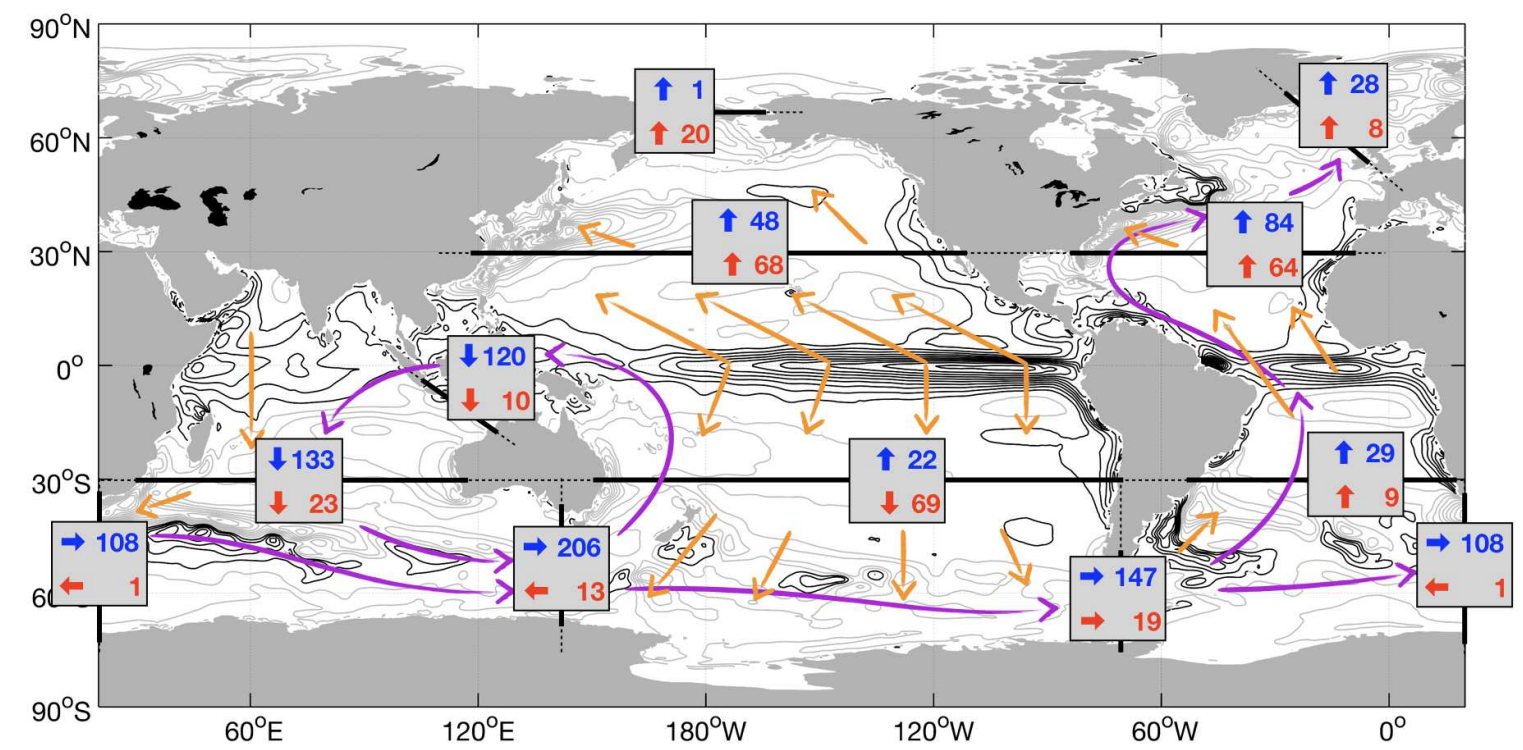

Figure 1: Ocean heat transport (OHT in $0.01 \mathrm{PW} ; 1 \mathrm{PW}=10^{15} \mathrm{~W}$ ) as estimated through gates that separate ocean basins and through meridians that delimit the tropics (thick black lines) for the 1992-2011 time mean. Raw OHT (rotational+divergent components) and effective OHT (divergent component only) values are charted in, respectively, blue and red. Thin lines with arrow heads are a schematic of the main internal oceanic heat loops (purple) and effective OHT patterns (orange). Black contours (resp. grey contours) represent the rate of ocean heat divergence (resp. convergence) which reflects the rate of heat uptake from (resp. heat release to) the atmosphere. These rates are contoured every $15 \mathrm{~W} / \mathrm{m}^{2}$ intervals starting from $\pm 5 \mathrm{~W} / \mathrm{m}^{2}$. 


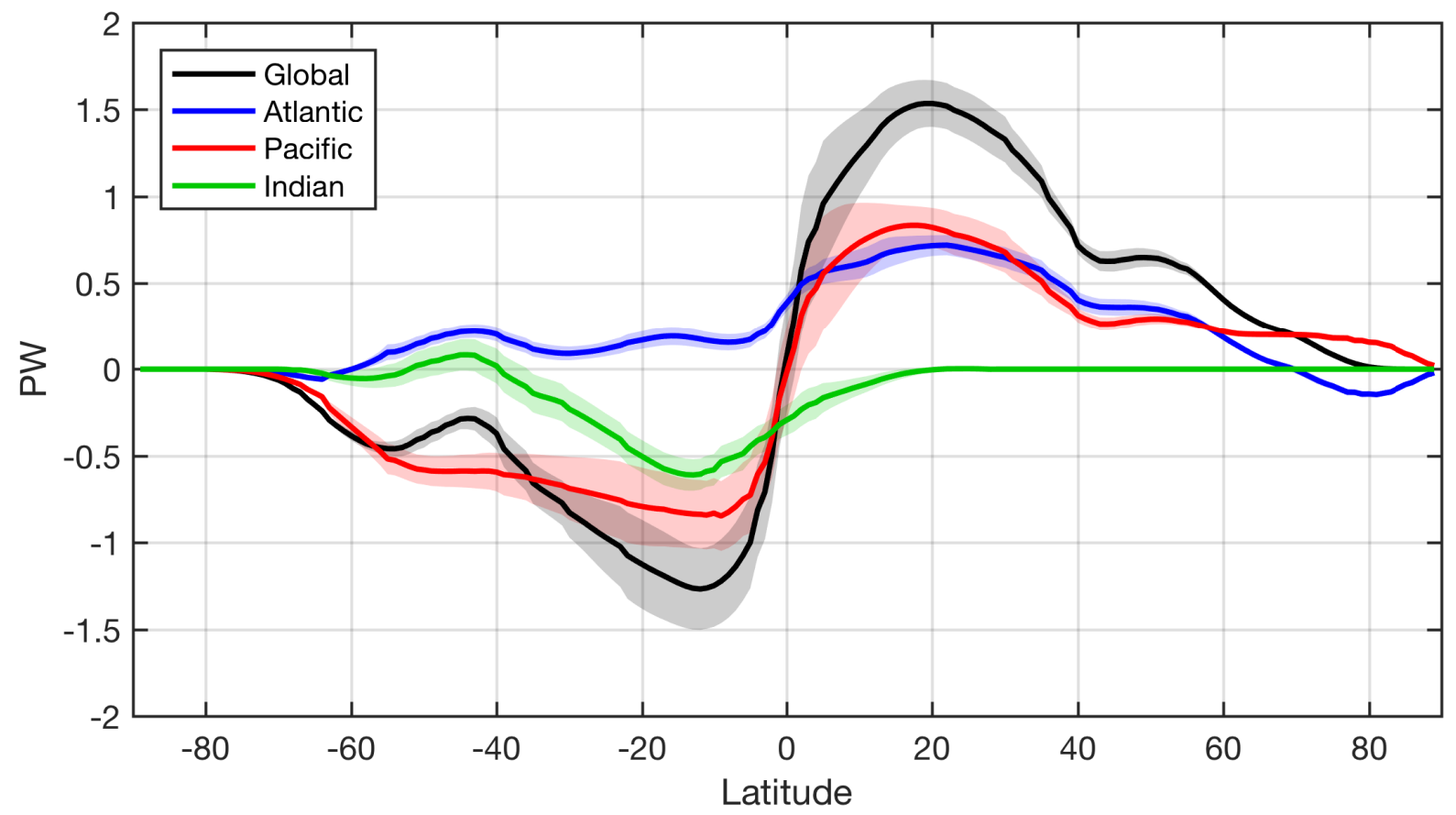

Figure 2: Meridional ocean heat transport (OHT; in PW) for the global ocean is depicted in black. Also shown: meridional effective OHT for the Atlantic (blue), Pacific (red), and Indian (green) sectors. Thick lines denote the 1992-2011 mean and shaded ranges reflect \pm 1 standard deviation amongst annual means. 


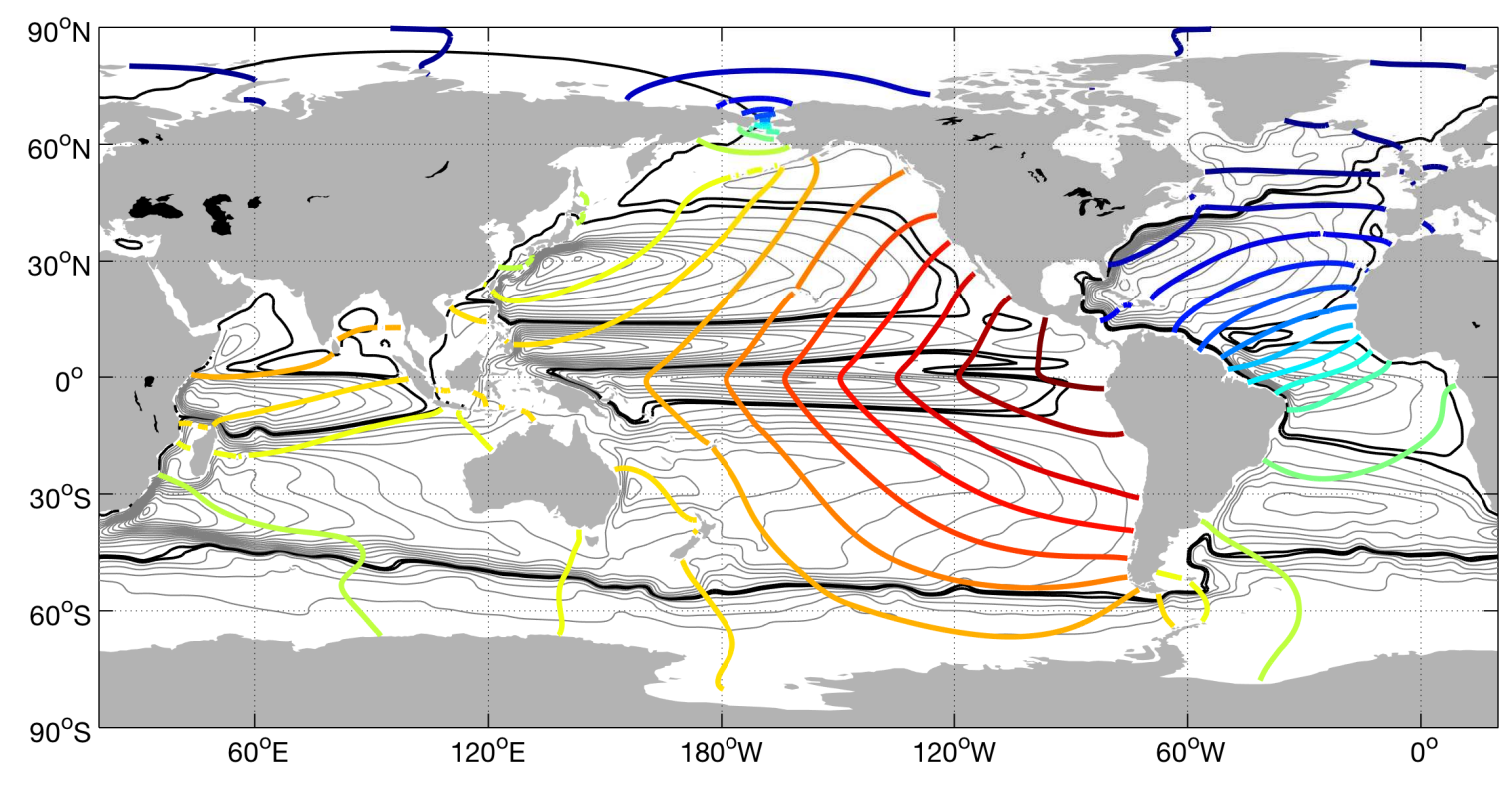

Figure 3: Scalar potential (colored contours; in $0.2 \mathrm{PW}$ intervals) and vector potential (black and grey contours; in 0.05 then $0.2 \mathrm{PW}$ intervals) as derived from the global vector field of rawOHT averaged over 1992-2011. The scalar and vector potentials determine, respectively, effOHT and rotOHT (see SPM, section 2). Each contour of the vector potential can be thought of as a heat loop confined within the ocean. In contrast, effOHT is oriented perpendicularly across contours of the scalar potential and directed from red to blue contours. 


\section{References}

1. T. F. Stocker, International Geophysics 103, 3 (2013). Ocean Circulation and Climate.

2. A. Ganachaud, C. Wunsch, Nature 408, 453 (2000).

3. K. E. Trenberth, J. M. Caron, Journal of Climate 14, 3433 (2001).

4. A. M. Macdonald, M. O. Baringer, Ocean Circulation and ClimateA 21st Century Perspective, J. G. Gerold Siedler, Stephen M. Griffies, J. A. Church, eds. (Academic Press, 2013), vol. 103 of International Geophysics, pp. $759-785$.

5. J. T. Fasullo, K. E. Trenberth, Journal of Climate 21, 2313 (2008).

6. K. E. Trenberth, J. T. Fasullo, Geophysical Research Letters 44, 1919 (2017). 2016GL072475.

7. K. Ds, et al., Journal of Climate 30, 631 (2017).

8. G. A. Schmidt, C. M. Bitz, U. Mikolajewicz, L.-B. Tremblay, Ocean Modelling 7, 59 (2004).

9. K. Bryan, Journal of Geophysical Research 67, 3403 (1962).

10. B. A. Warren, Journal of Geophysical Research: Oceans 104, 7915 (1999).

11. A. Ganachaud, C. Wunsch, Journal of Climate 16, 696 (2003).

12. W. E. Johns, et al., Journal of Climate 24, 2429 (2011).

13. L. D. Talley, Journal of Physical Oceanography 33, 530 (2003).

14. T.-C. Chen, Monthly Weather Review 113, 1801 (1985). 
15. I. G. Watterson, Journal of Atmospheric and Oceanic Technology 18, 691 (2001).

16. H. Corell, J. Nilsson, K. DS, G. Brostrm, Tellus A: Dynamic Meteorology and Oceanography 61, 635 (2008).

17. G. Forget, et al., Geoscientific Model Development 8, 3071 (2015).

18. G. Forget, D. Ferreira, X. Liang, Ocean Science 11, 839 (2015).

19. G. Forget, R. Ponte, Progress in Oceanography pp. 173-195 (2015).

20. G. Forget, et al. (2016).

21. D. G. Evans, et al., Journal of Physical Oceanography 0, null (2017).

22. M. Bowen, et al., Journal of Climate 30, 7481 (2017).

23. Q. Wu, X. Zhang, J. A. Church, J. Hu, Journal of Geophysical Research: Oceans 122, 1862 (2017).

24. B. Meyssignac, et al., Surveys in Geophysics 38, 187 (2017).

25. R. Lumpkin, K. Speer, Journal of Physical Oceanography 37, 2550 (2007).

26. G. Boccaletti, R. Ferrari, A. Adcroft, D. Ferreira, J. Marshall, Geophysical Research Letters 32, n/a (2005). L10603.

27. R. Ferrari, D. Ferreira, Ocean Modelling 38, 171 (2011).

28. A. C. Hirst, J. Godfrey, Journal of Physical Oceanography 23, 1057 (1993).

29. C. Sun, D. R. Watts, Journal of Geophysical Research: Oceans 107, 2 (2002). 3119.

30. V. Tamsitt, L. D. Talley, M. R. Mazloff, I. Ceroveki, Journal of Climate 29, 6563 (2016). 
31. R. A. Woodgate, T. Weingartner, R. Lindsay, Geophysical Research Letters 37, n/a (2010). L01602.

32. J. S. Godfrey, Geophysical \& Astrophysical Fluid Dynamics 45, 89 (1989).

33. K. Speer, G. Forget, Ocean Circulation and Climate: A 21 st century perspective, International Geophysics (Elsevier Science, Amsterdam, Netherlands, 2013), chap. Global Distribution and Formation of Mode Waters, pp. 211-226.

34. M. Balmaseda, et al., Journal of Operational Oceanography 8, s80 (2015). 


\title{
Supplementary Material For Effective Ocean Heat Transport
}

\author{
Gaël Forget, ${ }^{1 *}$ David Ferreira, ${ }^{2}$ \\ ${ }^{1}$ Dept. of Earth, Atmospheric and Planetary Sciences, \\ Massachusetts Institute of Technology, Cambridge, MA 02139, USA. \\ ${ }^{2}$ University of Reading, Department of Meteorology, \\ PO box 243, Reading, RG6 6BB, UK.
}

*To whom correspondence should be addressed; E-mail: gforget@mit.edu.

\section{Ocean State Estimate}

2 Our analysis is based on the ECCO version 4 release 2 gridded data product $(1,2)$. The ECCO v4 ocean state estimate agrees, within expected uncertainty ranges, with the bulk of available ocean observations as well as with atmospheric re-analysis data over 1992-2011. It includes a full set of optimized transport estimates across the sea surface and in the ocean interior along with recent estimate of geothermal heat fluxes (3) applied at the sea floor. The chosen, adjointbased estimation methodology does not rely on assimilation increments (4) but rather optimizes surface forcing fields and internal model parameters $(1,5,6)$. As a result, the ECCO v4 product offers closed heat budgets where the convergence of transports matches the ocean state evolution to machine precision.

The underlying configuration of the MIT general circulation model $(1,7)$ uses the "real freshwater flux" formulation, accounting for mass fluxes across the sea surface associated with precipitation, evaporation, run off, and sea ice freezing and melting. However, as commonly 
done in climate modelling, the model makes the Boussinesq approximation and neglects specific heat capacity variations $(1,5)$. In practice, this means that for any given volume element $\mathrm{V}$ the corresponding heat content is simply given by $\mathrm{H}=\rho_{c} C_{p} \theta \mathrm{V}$ where $\rho_{c}$ is the constant Boussinesq density, $C_{p}$ the constant specific heat capacity, and $\theta$ the potential temperature averaged over V. Accordingly, if $\mathbf{U}$ denotes a volume flux across the sea surface or in the ocean interior then the corresponding mass flux is given by $\mathbf{M}=\rho_{c} \mathbf{U}$. In this paper, we follow common oceanographic usage by referring to $\rho_{c} C_{p} \theta \mathbf{U}$ simply as heat transport rather than as approximation of total energy transport which would be more rigorous $(8,9)$.

All variables needed for the presented analyses are permanently archived $(10,11)$ and readily available for download via the ECCO website (http://ecco-group.org/).

\section{Computation of the divergent and rotational transports}

First, we sum up all monthly-averaged advective and diffusive transports of potential temperature (10), multiply them by $\rho_{c} C_{p}$, integrate vertically, and form annual averages from 1992 to 2011. The resulting vector fields are referred to as raw $\mathrm{OHT}$ in this article and as $\mathbf{F}$ in equations reported below. As is classically done in Helmholtz decomposition, we then separate $\mathbf{F}$ into a divergent component, $\mathbf{F}_{d i v}$, and a rotational component, $\mathbf{F}_{\text {rot }}$ :

$$
\mathbf{F}=\mathbf{F}_{d i v}+\mathbf{F}_{r o t}
$$

satisfying:

$$
\nabla \times \mathbf{F}_{d i v}=0 \text { and } \nabla \cdot \mathbf{F}_{r o t}=0
$$

These components are associated with a scalar potential, $\mathcal{P}$, and a streamfunction, $\mathcal{S}$, such that:

$$
\begin{aligned}
& \mathbf{F}_{\text {div }}=\nabla \mathcal{P} \\
& \mathbf{F}_{\text {rot }}=\nabla \times \mathcal{S} .
\end{aligned}
$$


In practice, the scalar potential, $\mathcal{P}$, is computed by solving, over the global ocean, the Poisson equation:

$$
\nabla^{2} \mathcal{P}=\nabla \cdot \mathbf{F}
$$

obtained by taking the divergence of Eq. (1) and using Eqs. (2-3). The divergent component, $\mathbf{F}_{d i v}$, is then readily derived from $\mathcal{P}$ using Eq. (3); it is referred to as effOHT in this article. Finally, the rotational component is computed as the remainder, $\mathbf{F}_{r o t}=\mathbf{F}-\mathbf{F}_{d i v}$, and integration of its transverse component along grid line paths readily gives $\mathcal{S}$.

The Poisson equation is formulated such that $\mathbf{F}_{\text {div }}$ obeys a zero normal flow boundary condition at the coastline over the global Ocean. This choice makes the most immediate physical and practical sense as argued by (12). It is indeed consistent with the underlying zero normal flow boundary condition for $\mathbf{F}$ in the ocean model, so that the rotational component, $\mathbf{F}_{r o t}=\mathbf{F}-\mathbf{F}_{d i v}$, also satisfies a zero normal flow boundary condition. Hence, $\mathbf{F}, \mathbf{F}_{d i v}$, and $\mathbf{F}_{r o t}$ all are tangential to the coastline, and the decomposition of $\mathbf{F}$ as $\mathbf{F}_{d i v}+\mathbf{F}_{r o t}$ yields a simple extension of the familiar notion of meridional transports bounded by coastlines (Fig. S1).

The 20 -year mean $\mathcal{P}$ and $\mathcal{S}$ are depicted in Fig. 3 as, respectively, colored and grey contours. The raw and effective transports reported in Figs. 1 - 2 and Tabs. S1 - S4 are computed by integrating the transverse component of either $\mathbf{F}_{d i v}$ or $\mathbf{F}$ following grid line paths that are shown in Fig. S2. Algorithmic detail is provided in appendix C of (1). When computing pole to pole meridional transports for the Atlantic, Pacific, and Indian oceans, the Arctic is split into two sectors along the $80^{\circ} \mathrm{W}$ and $100^{\circ} \mathrm{E}$ meridians, the Southern Ocean is split into three sectors along Drake Passage, $147^{\circ} \mathrm{E}$, and $20^{\circ} \mathrm{W}$, and marginal seas are attached to neighboring oceans as reflected by the color coding in Fig. S2. 


\section{Underlying physical Processes}

To evaluate uncertainties associated with our interpretation of effOHT, it is useful to consider the various terms of the temperature equation computed at constant volume (11). The underlying heat budget can be written for a time-variable volume element, $\mathrm{V}$, as

$$
\frac{d \mathrm{H}}{d t}=\rho_{c} C_{p}\left(\theta \frac{d \mathrm{~V}}{d t}+\frac{d \theta}{d t} \mathrm{~V}\right)
$$

where $\rho_{c} \frac{d \mathrm{~V}}{d t}$ is the rate of mass flux convergence and $\frac{d \theta}{d t}$ is the rate of temperature change within the selected volume element. Detailed equations are provided in (1).

The temperature equation computed at constant volume, which by construction is free of any mass-flux convergence, expresses the second term on the right hand side of Eq. (6) as

$$
\rho_{c} C_{p} \mathrm{~V} \frac{d \theta}{d t}=h_{t e n d}=h_{a d v}+h_{d i f}+h_{\text {forc }}
$$

where $h_{a d v}$ represents the advective heat flux convergence, $h_{d i f}$ the diffusive heat flux convergence, $h_{\text {forc }}$ the external forcing due to air-sea and geothermal heat flux convergence, and $h_{\text {tend }}$ the temperature tendency term. Any term in Eq. (7), once its global mean has been removed, can be substituted with $\nabla \cdot \mathbf{F}$ on the right-hand-side of the Poisson equation (5) to translate it into a divergent vector field associated with a scalar potential like in Eq. (3).

A close approximation to effOHT is obtained by applying this approach to $h_{a d v}+h_{d i f}$ (e.g., compare Fig. S3 with Fig. 2) since the impact of non-zero mass fluxes on effOHT is generally small (SPM section 4). The residual (i.e., the difference between Fig. S3 and Fig. 2) shows little interannual variability and time-mean values of the order of $\pm 0.1 \mathrm{PW}$ in terms of meridional transports (Fig. S4; left) with patterns that resemble those seen in net mass transports (2).

Further unpacking the terms in Eq. (7) reveals that effOHT primarily reflects a balance between advection and air-sea fluxes (top panels in Fig. S5). Lateral diffusion contributes $\pm 0.2 \mathrm{PW}$ in the time mean with negligible interannual variability (bottom left) while temporal variations in ocean temperature translate into negligible effOHT for the 20 -year average 
along with $\pm 0.2 \mathrm{PW}$ interannual variability in the tropics (bottom right). As a result, indirect estimates of annual-mean meridional heat transports based on air-sea flux maps can only approximate effOHT at best within $\pm 0.3 \mathrm{PW}$. Such uncertainty levels are unfortunately comparable to the magnitude of effective inter-ocean heat transports (Tab. S1).

\section{Sensitivity Tests}

The approach introduced in SPM sections 1-2-3 has noteworthy advantages as compared with previous methods. Unlike earlier estimations of the divergent OHT component in (13) and similar studies, it [a] addresses also the non-divergent OHT component, [b] readily filters out global imbalances which may come from atmospheric data assimilation schemes or anthropogenic heat uptake $(4,14),[c]$ ensures strict consistency between air-sea fluxes, ocean heat content changes, and $\operatorname{OHT}(1,2)$, and [d] avoids ad-hoc integration method or boundary condition choices in deriving meridional transports. As compared with ship-based estimations, our approach further [e] alleviates uncertainty associated with energy reference level choices and non-zero mass fluxes $(15,16)$. Issues [d] and [e] remain to be illustrated, which is done below.

\subsection{Sensitivity to the integration method}

First, we replicate the method of (13) to infer meridional OHTs by cumulatively integrating $\nabla \cdot \mathbf{F}$ from the North pole to the South pole over the Atlantic, Pacific, and Indian. Similar methods have been used to infer meridional OHTs from air-sea flux maps going back at least as far as the 1950's (17). Closely following the methodology of (13) facilitates comparison with their Fig. 3. Their methodological choices imply that OHTs through the Indonesian Archipelago and through the Southern Hemisphere passages between the Atlantic, Pacific, and Indian are set to zero. Still following (13), the whole Arctic is attached to the Atlantic in this computation, which de facto also sets OHT through Bering Strait to zero. The result of this computation is hereafter 
denoted by infOHT and is depicted in Fig. S6 (dashed curves).

The ECCO estimate of infOHT (Fig. S6; dashed curves) is in good agreement with the result shown in Fig. 3 of (13), which is based on a combination of top-of-atmosphere radiation estimates with atmospheric and oceanic reanalyses products. However, comparison between our infOHT (Fig. S6; dashed curves) and our effOHT (Fig. S6; solid curves), both based on the exact same ECCO estimate of $\nabla \cdot \mathbf{F}$, reveals biases in infOHT.

Starting from the North Pole, the closing of Bering Strait leads to a northward OHT bias in the Atlantic and a corresponding southward OHT bias in the Pacific. In turn, the ITF closing induces a northward OHT bias in the Indian while adding southward OHT bias south of $\sim 10^{\circ} \mathrm{S}$ in the Pacific. Additional compounding of OHT bias occurs further South as a result of closing Drake Passage and the section between Australia and Antarctica.

Once integration has reached the coast of Antarctica, the end result is an unbalanced OHT of $0.39,-0.62$, and $0.22 \mathrm{PW}$ for the Atlantic, Pacific, and Indian respectively. These large imbalances are consistent with those reported in (13), and result from introducing artificial boundaries of integration between oceanic basins. As already pointed out by (13), this clearly argues in favor of a more objective approach such as the one introduced in this paper.

\subsection{Sensitivity to the Energy Reference Level}

Now, we assess the sensitivity of OHT estimates to arbitrary ERL choices, which can be a major concern in the case of ship-based OHT estimates due to non-zero mass fluxes $(15,16)$. To assess the impact of ERL choices, we subtract $\rho_{c} C_{p} \theta_{0} \mathbf{U}$, where $\theta_{0}$ is a uniform temperature value and $\rho_{c} \mathbf{U}$ is the ECCO mass-transport estimate, from $\mathbf{F}$ and recompute $\mathbf{F}_{d i v}$ and $\mathbf{F}_{r o t}$ via Eqs. 3-4-5. It should be noted that $\rho_{c} \mathbf{U}$ itself includes both rotational and divergent components. Results shown previously in Figs. 1 to 3, Figs. S2 to S6, and Tabs S1 to S4 correspond to $\theta_{0}=0^{\circ} \mathrm{C}$. Here we test two other reasonable choices for $\theta_{0}: 3.5^{\circ} \mathrm{C}$ and $18.5^{\circ} \mathrm{C}$ which correspond to setting 
the ERL approximately to, respectively, the global mean ocean temperature and the global mean sea surface temperature.

Comparing results from the $3.5^{\circ} \mathrm{C}$ and $18.5^{\circ} \mathrm{C}$ cases reveals that rawOHT is often very sensitive to ERL choices whereas effOHT is much more robust (e.g., compare Tabs. S5 with S6). This contrast in sensitivity between rawOHT and effOHT reflects that rotational mass flux magnitudes associated with gyres, the Antarctic Circumpolar Current, etc. vastly exceed divergent mass flux magnitudes associated with evaporation and precipitation $(1,2)$. As a result, the OHT streamfunction $\mathcal{S}$ associated, per Eq. (4), with the rotational flux $\mathbf{F}_{\text {rot }}$ differs wildly between the two panels of Fig. S7 as well as between Fig. S7 and Fig. 3. In contrast, the OHT scalar potential $\mathcal{P}$ is remarkably insensitive to the choices of ERL: effOHT changes by less than 0.01-0.02 PW in these tests, an effect well below other sources of uncertainties (Tabs. S5 and S6).

In conclusion, even the sign and order of magnitude of rawOHT should be interpreted with great caution as they are essentially predicated on an arbitrary ERL choice. As a consequence, heterogeneous treatment of non-zero mass fluxes can, in and of itself, greatly complicate OHT estimate inter-comparisons. 
Table S1: Effective and raw ocean heat transports (in PW; $1 \mathrm{PW}=10^{15} \mathrm{~W}$ ) computed through passages that separate the main oceanic basins (red sections in Fig. S2). The top four values (resp. bottom five) are counted positive northward (resp. eastward). Reported values are for the 1992-2011 mean \pm 1 standard deviation amongst annual means.

\begin{tabular}{||c|c||c||}
\hline \hline $\begin{array}{c}\text { effective transport } \\
\text { effOHT }\end{array}$ & $\begin{array}{c}\text { raw transport } \\
\text { rawOHT }\end{array}$ & section name \\
\hline \hline$-0.13 \pm 0.06$ & $+2.06 \pm 0.03$ & Australia-Antarctica $\left(147^{\circ} \mathrm{E}\right)$ \\
$+0.19 \pm 0.07$ & $+1.47 \pm 0.02$ & Drake Passage \\
$-0.01 \pm 0.06$ & $+1.08 \pm 0.03$ & South Africa-Antarctica $\left(19.5^{\circ} \mathrm{W}\right)$ \\
\hline$-0.10 \pm 0.11$ & $-1.20 \pm 0.10$ & Indonesian Through Flow \\
& & Bering Strait \\
& (negative values = westward transport) \\
\hline$+0.20 \pm 0.02$ & $+0.01 \pm 0.00$ & Davis Strait \\
$-0.01 \pm 0.00$ & $+0.01 \pm 0.00$ & Denmark Strait \\
$+0.02 \pm 0.00$ & $+0.01 \pm 0.00$ & Iceland-Scotland \\
$+0.06 \pm 0.01$ & $+0.26 \pm 0.02$ & Scotland-Norway \\
$+0.01 \pm 0.00$ & $-0.00 \pm 0.00$ & \\
\hline \hline
\end{tabular}


Table S2: Effective and raw ocean heat transports (in PW) across meridians over the Indian Ocean (green sections in Fig. S2). All values are counted positive northward. Reported values are for the 1992-2011 mean \pm 1 standard deviation amongst annual means.

\begin{tabular}{||c|c||c||}
\hline \hline $\begin{array}{c}\text { effective transport } \\
\text { effOHT }\end{array}$ & $\begin{array}{c}\text { raw transport } \\
\text { rawOHT }\end{array}$ & Latitude \\
\hline$-0.00 \pm 0.01$ & $-0.00 \pm 0.01$ & $20^{\circ} \mathrm{N}$ \\
$-0.10 \pm 0.05$ & $-0.10 \pm 0.05$ & $10^{\circ} \mathrm{N}$ \\
$-0.29 \pm 0.09$ & $-0.33 \pm 0.08$ & Equator \\
$-0.58 \pm 0.09$ & $-1.04 \pm 0.10$ & $10^{\circ} \mathrm{S}$ \\
$-0.50 \pm 0.09$ & $-1.60 \pm 0.13$ & $20^{\circ} \mathrm{S}$ \\
$-0.23 \pm 0.11$ & $-1.33 \pm 0.09$ & $30^{\circ} \mathrm{S}$ \\
$+0.02 \pm 0.10$ & $+0.34 \pm 0.08$ & $40^{\circ} \mathrm{S}$ \\
$+0.03 \pm 0.08$ & $-1.79 \pm 0.08$ & $50^{\circ} \mathrm{S}$ \\
$-0.05 \pm 0.04$ & $-0.26 \pm 0.01$ & $60^{\circ} \mathrm{S}$ \\
\hline \hline
\end{tabular}

Table S3: Same as Table S2 but for the Pacific Ocean (blue sections in Fig. S2).

\begin{tabular}{||c|c||c||}
\hline \hline $\begin{array}{c}\text { effective transport } \\
\text { effOHT }\end{array}$ & $\begin{array}{c}\text { raw transport } \\
\text { rawOHT }\end{array}$ & Latitude \\
\hline$+0.16 \pm 0.02$ & $-0.01 \pm 0.00$ & $80^{\circ} \mathrm{N}$ \\
$+0.20 \pm 0.02$ & $+0.00 \pm 0.00$ & $70^{\circ} \mathrm{N}$ \\
$+0.22 \pm 0.02$ & $+0.02 \pm 0.00$ & $60^{\circ} \mathrm{N}$ \\
$+0.29 \pm 0.03$ & $+0.10 \pm 0.02$ & $50^{\circ} \mathrm{N}$ \\
$+0.31 \pm 0.04$ & $+0.12 \pm 0.03$ & $40^{\circ} \mathrm{N}$ \\
$+0.68 \pm 0.12$ & $+0.48 \pm 0.11$ & $30^{\circ} \mathrm{N}$ \\
$+0.82 \pm 0.11$ & $+0.63 \pm 0.10$ & $20^{\circ} \mathrm{N}$ \\
$+0.73 \pm 0.23$ & $+0.54 \pm 0.24$ & $10^{\circ} \mathrm{N}$ \\
$-0.02 \pm 0.27$ & $-0.18 \pm 0.29$ & Equator \\
$-0.83 \pm 0.20$ & $-0.56 \pm 0.17$ & $10^{\circ} \mathrm{S}$ \\
$-0.79 \pm 0.22$ & $+0.11 \pm 0.14$ & $20^{\circ} \mathrm{S}$ \\
$-0.69 \pm 0.18$ & $+0.22 \pm 0.08$ & $30^{\circ} \mathrm{S}$ \\
$-0.59 \pm 0.11$ & $+0.30 \pm 0.06$ & $40^{\circ} \mathrm{S}$ \\
$-0.58 \pm 0.08$ & $+0.37 \pm 0.10$ & $50^{\circ} \mathrm{S}$ \\
$-0.33 \pm 0.06$ & $-0.17 \pm 0.03$ & $60^{\circ} \mathrm{S}$ \\
$-0.05 \pm 0.00$ & $-0.05 \pm 0.00$ & $70^{\circ} \mathrm{S}$ \\
\hline \hline
\end{tabular}


Table S4: Same as Table S2 but for the Atlantic Ocean (yellow sections in Fig. S2).

\begin{tabular}{||c|c||c||}
\hline \hline $\begin{array}{c}\text { effective transport } \\
\text { effOHT }\end{array}$ & $\begin{array}{c}\text { raw transport } \\
\text { rawOHT }\end{array}$ & Latitude \\
\hline$-0.14 \pm 0.02$ & $+0.02 \pm 0.00$ & $80^{\circ} \mathrm{N}$ \\
$-0.01 \pm 0.02$ & $+0.19 \pm 0.02$ & $70{ }^{\circ} \mathrm{N}$ \\
$+0.18 \pm 0.02$ & $+0.38 \pm 0.02$ & $60{ }^{\circ} \mathrm{N}$ \\
$+0.35 \pm 0.04$ & $+0.55 \pm 0.03$ & $50{ }^{\circ} \mathrm{N}$ \\
$+0.40 \pm 0.05$ & $+0.60 \pm 0.04$ & $40^{\circ} \mathrm{N}$ \\
$+0.65 \pm 0.06$ & $+0.84 \pm 0.06$ & $30{ }^{\circ} \mathrm{N}$ \\
$+0.72 \pm 0.06$ & $+0.91 \pm 0.07$ & $20^{\circ} \mathrm{N}$ \\
$+0.61 \pm 0.08$ & $+0.81 \pm 0.09$ & $10^{\circ} \mathrm{N}$ \\
$+0.39 \pm 0.05$ & $+0.58 \pm 0.06$ & Equator \\
$+0.16 \pm 0.05$ & $+0.36 \pm 0.06$ & $10^{\circ} \mathrm{S}$ \\
$+0.17 \pm 0.05$ & $+0.36 \pm 0.06$ & $20^{\circ} \mathrm{S}$ \\
$+0.09 \pm 0.04$ & $+0.29 \pm 0.04$ & $30^{\circ} \mathrm{S}$ \\
$+0.20 \pm 0.04$ & $-1.01 \pm 0.06$ & $40^{\circ} \mathrm{S}$ \\
$+0.16 \pm 0.04$ & $+1.03 \pm 0.03$ & $50^{\circ} \mathrm{S}$ \\
$-0.00 \pm 0.02$ & $+0.04 \pm 0.01$ & $60^{\circ} \mathrm{S}$ \\
$-0.02 \pm 0.00$ & $-0.02 \pm 0.00$ & $70^{\circ} \mathrm{S}$ \\
\hline \hline
\end{tabular}


Table S5: Transports as in Tabs. S1 to S4 (in PW) but with the Energy Reference Level (ERL) set to the global mean ocean temperature $\left(3.5^{\circ} \mathrm{C}\right)$. See SPM section 4 for detail.

\begin{tabular}{||c|c||c||}
\hline \hline $\begin{array}{c}\text { effective transport } \\
\text { effOHT }\end{array}$ & $\begin{array}{c}\text { raw transport } \\
\text { rawOHT }\end{array}$ & section name \\
\hline \hline$-0.13 \pm 0.06$ & $-0.34 \pm 0.03$ & Australia-Antarctica $\left(147^{\circ} \mathrm{E}\right)$ \\
$+0.19 \pm 0.07$ & $-0.70 \pm 0.04$ & Drake Passage \\
$-0.01 \pm 0.06$ & $-1.10 \pm 0.05$ & South Africa-Antarctica $\left(19.5^{\circ} \mathrm{W}\right)$ \\
\hline$-0.10 \pm 0.11$ & $-0.98 \pm 0.09$ & Indonesian Through Flow \\
\hline$+0.20 \pm 0.02$ & $-0.01 \pm 0.00$ & Bering Strait \\
\hline$-0.29 \pm 0.09$ & $-0.33 \pm 0.08$ & Equator (Indian) \\
$-0.23 \pm 0.11$ & $-1.12 \pm 0.09$ & $-30 \mathrm{~N}$ (Indian) \\
\hline$+0.68 \pm 0.12$ & $+0.47 \pm 0.11$ & $+30 \mathrm{~N}$ (Pacific) \\
$-0.01 \pm 0.27$ & $-0.19 \pm 0.29$ & Equator (Pacific) \\
$-0.69 \pm 0.18$ & $-0.02 \pm 0.08$ & $-30 \mathrm{~N}$ (Pacific) \\
\hline$+0.65 \pm 0.06$ & $+0.86 \pm 0.06$ & $+30 \mathrm{~N}$ (Atlantic) \\
$+0.39 \pm 0.05$ & $+0.60 \pm 0.06$ & Equator (Atlantic) \\
$+0.09 \pm 0.04$ & $+0.30 \pm 0.04$ & $-30 \mathrm{~N}$ (Atlantic) \\
\hline \hline
\end{tabular}

Table S6: Transports as in Tabs. S1 to S4 (in PW) but with the Energy Reference Level (ERL) set to the global mean sea surface temperature $\left(18.5^{\circ} \mathrm{C}\right)$. See SPM section 4 for detail.

\begin{tabular}{||c|c||c||}
\hline \hline $\begin{array}{c}\text { effective transport } \\
\text { effOHT }\end{array}$ & $\begin{array}{c}\text { raw transport } \\
\text { rawOHT }\end{array}$ & section name \\
\hline \hline$-0.12 \pm 0.06$ & $-10.62 \pm 0.20$ & Australia-Antarctica $\left(147^{\circ} \mathrm{E}\right)$ \\
$+0.19 \pm 0.07$ & $-10.01 \pm 0.19$ & Drake Passage \\
$-0.00 \pm 0.06$ & $-10.47 \pm 0.20$ & South Africa-Antarctica $\left(19.5^{\circ} \mathrm{W}\right)$ \\
\hline$-0.08 \pm 0.11$ & $-0.05 \pm 0.07$ & Indonesian Through Flow \\
\hline$+0.20 \pm 0.02$ & $-0.07 \pm 0.02$ & Bering Strait \\
\hline$-0.29 \pm 0.09$ & $-0.31 \pm 0.08$ & Equator (Indian) \\
$-0.24 \pm 0.11$ & $-0.21 \pm 0.08$ & $-30 \mathrm{~N}$ (Indian) \\
\hline$+0.70 \pm 0.12$ & $+0.43 \pm 0.11$ & $+30 \mathrm{~N}$ (Pacific) \\
$+0.02 \pm 0.26$ & $-0.23 \pm 0.28$ & Equator (Pacific) \\
$-0.71 \pm 0.18$ & $-1.01 \pm 0.12$ & $-30 \mathrm{~N}$ (Pacific) \\
\hline$+0.66 \pm 0.06$ & $+0.94 \pm 0.06$ & $+30 \mathrm{~N}$ (Atlantic) \\
$+0.40 \pm 0.05$ & $+0.67 \pm 0.06$ & Equator (Atlantic) \\
$+0.07 \pm 0.04$ & $+0.35 \pm 0.04$ & $-30 \mathrm{~N}$ (Atlantic) \\
\hline \hline
\end{tabular}




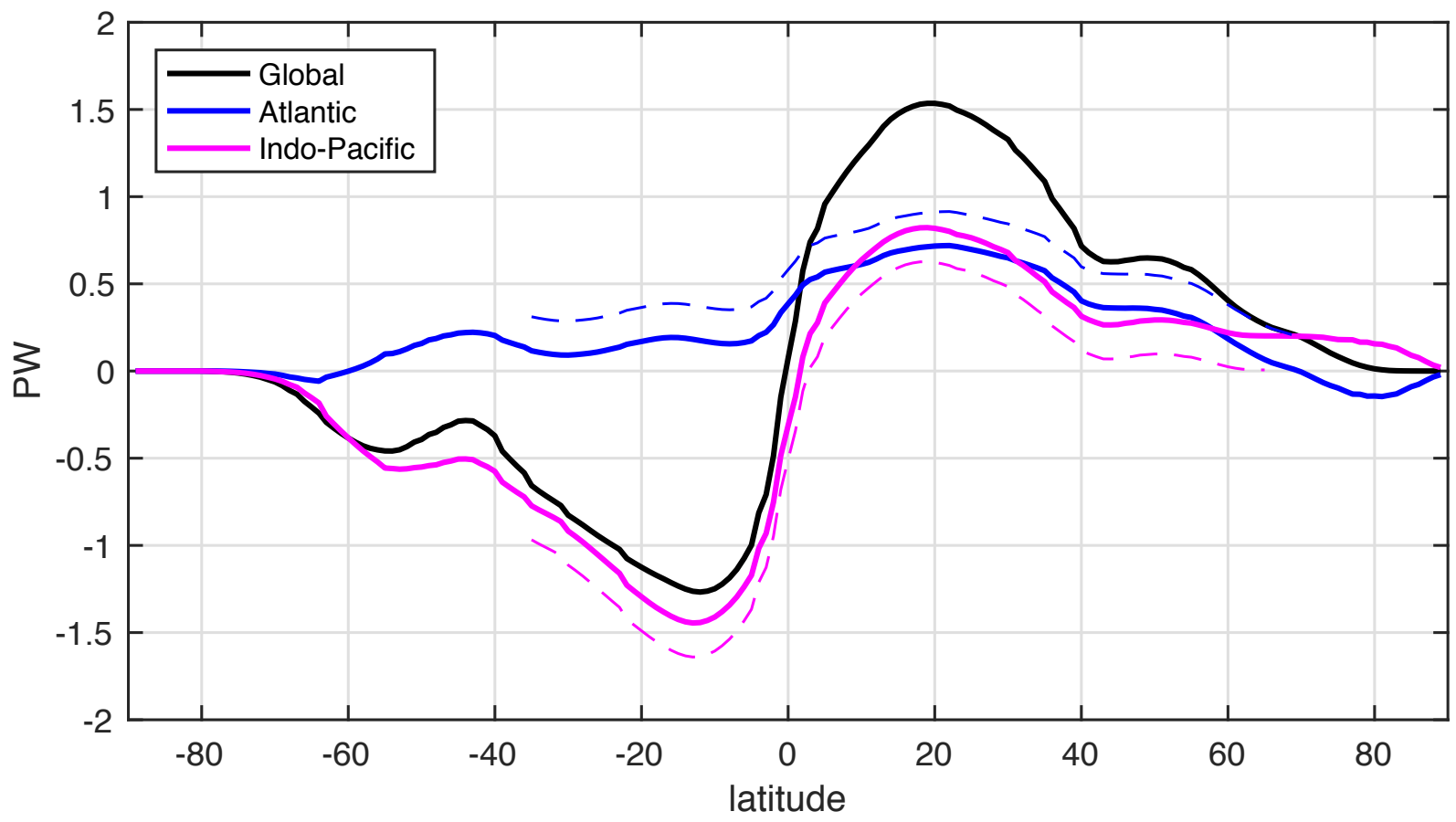

Figure S1: Meridional ocean heat transport (in PW; $1 \mathrm{PW}=10^{15} \mathrm{~W}$ ) on average over 1992-2011 for the Atlantic (blue) and the Indo-Pacific (red) based on either $\mathbf{F}_{d i v}$ (solid) or $\mathbf{F}$ (dashed). Net meridional transports integrated around the Globe are the same in both cases (black). 


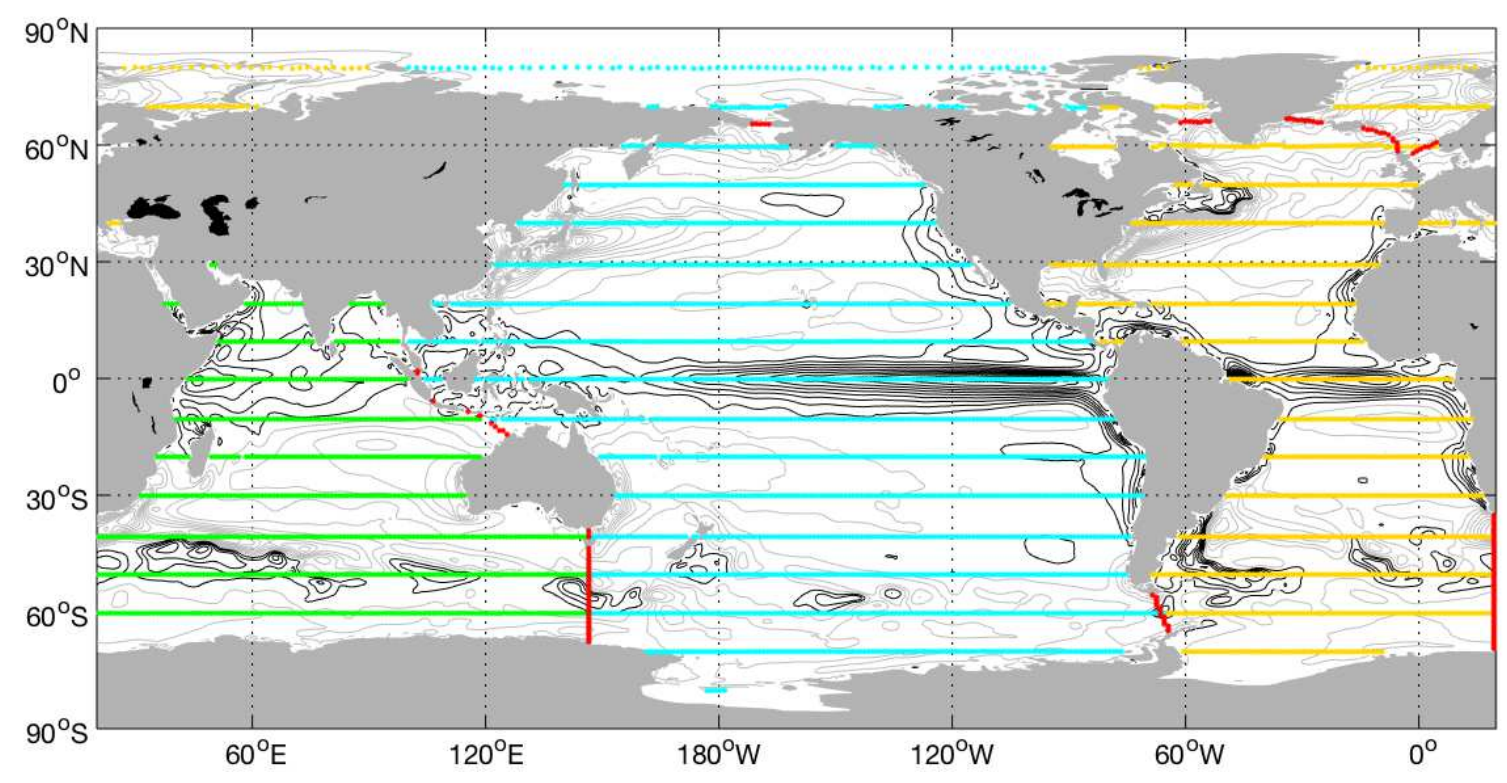

Figure S2: Transects through which heat transport estimates are reported in Tab. S1 (red transects), Tab. S2 (green transects), Tab. S3 (blue transects), and Tab. S4 (yellow transects). Black contours (resp. grey contours) represent the rate of ocean heat divergence (resp. convergence) which reflects the rate of heat uptake from (resp. heat release to) the atmosphere. 


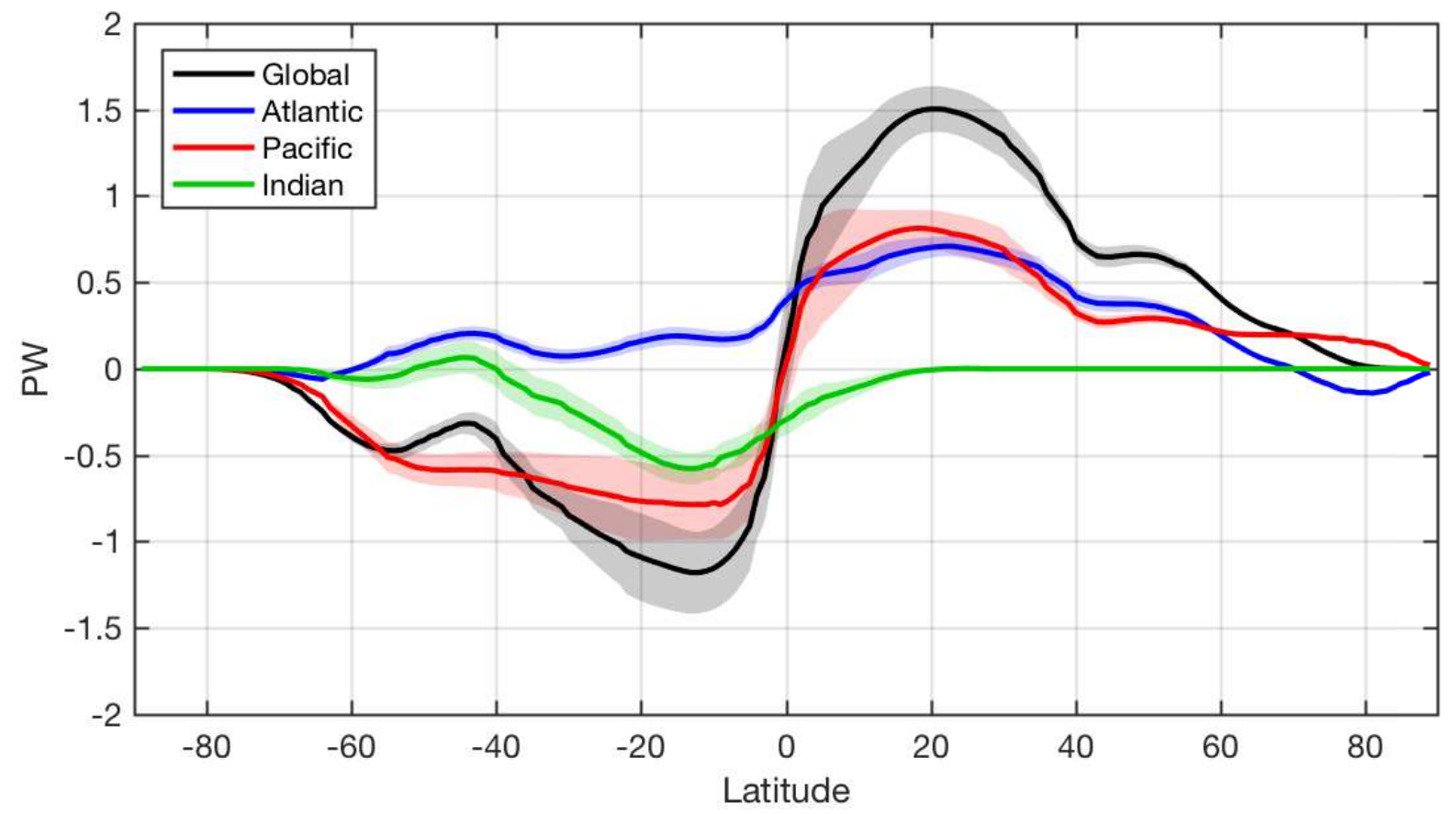

Figure S3: Implied meridional ocean transports (in PW) computed from the convergence of advective and diffusive fluxes $\left(h_{a d v}+h_{d i f}\right.$ in Eq. (7)) for the global ocean or separately for the Atlantic, Pacific, and Indian sectors. Unlike in Fig. 2, these results are based on the temperature tendency equation computed at constant volume: $h_{a d v}+h_{d i f}=h_{\text {tend }}-h_{\text {forc }}$ where $h_{\text {tend }}$ and $h_{\text {forc }}$ respectively represent the tendency and external forcing terms. Implied transports are computed from $h_{a d v}+h_{\text {dif }}$ by subtracting the global mean value and then solving a Poisson equation as done before for effOHT (Fig. 2). Thick lines denote the 1992-2011 mean and shaded ranges reflect \pm 1 standard deviation amongst annual means. 

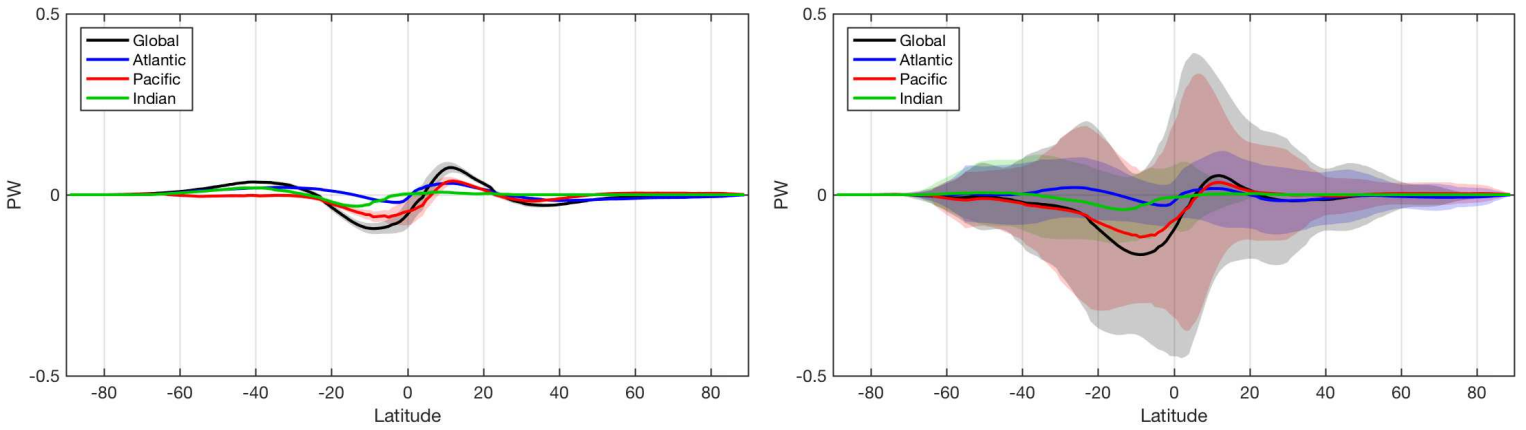

Figure S4: Left: difference between effOHT (Fig. 2) and meridional heat transports implied by $h_{a d v}+h_{d i f}$ in Eq. (7) (Fig. S3); this gives the residual contribution of convergent mass fluxes associated with the interplay of evaporation, precipitation, and runoff to effOHT. Right: difference between effOHT (Fig. 2) and meridional heat transports implied by $-h_{\text {forc }}$ in Eq. (7) (top right panel of Fig. S5); this corresponds to errors that would result from using air-sea heat fluxes as an estimate of ocean heat transport convergences. Thick lines denote the 1992-2011 mean and shaded ranges reflect \pm 1 standard deviation amongst annual means. 

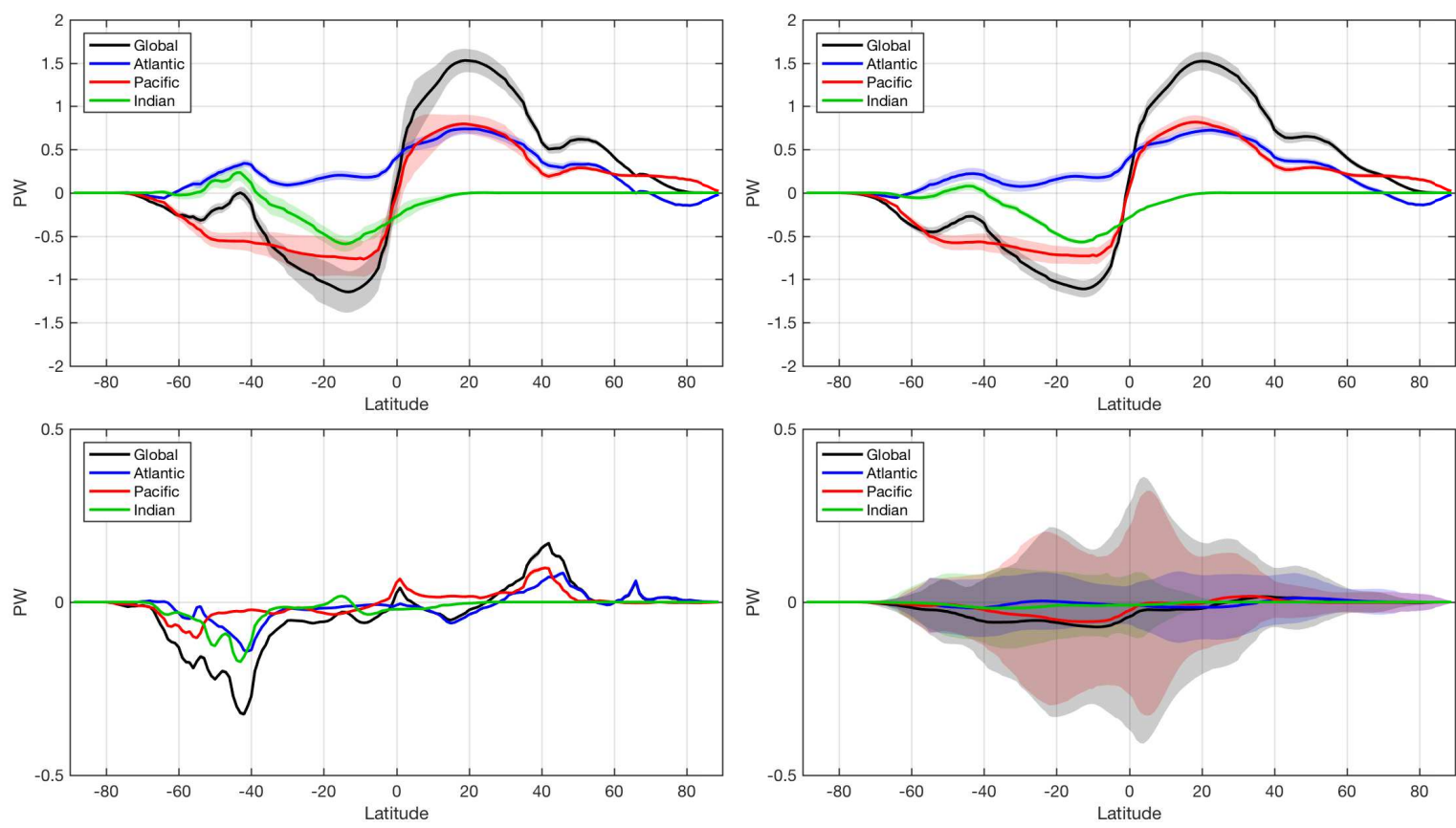

Figure S5: Implied meridional ocean heat transports (in PW) for each term of the temperature equation computed at constant volume (Eq. (7)). In each case, implied transports are computed by subtracting the global mean value and then solving a Poisson equation: (top left) the advective flux convergence $h_{a d v}$, (bottom left) the diffusive flux convergence $h_{d i f}$, (top right ) the vertical divergence of heat fluxes through the sea surface and sea floor $-h_{f o r c}$, and (bottom right) the tendency term $h_{\text {tend }}$. Note that, by construction, the sum of the left panels $\left(h_{a d v}+h_{d i f}\right)$ is equal to the sum of the right panels $\left(h_{\text {tend }}-h_{\text {forc }}\right)$. Thick lines denote the 1992-2011 mean and shaded ranges reflect \pm 1 standard deviation amongst annual means. 


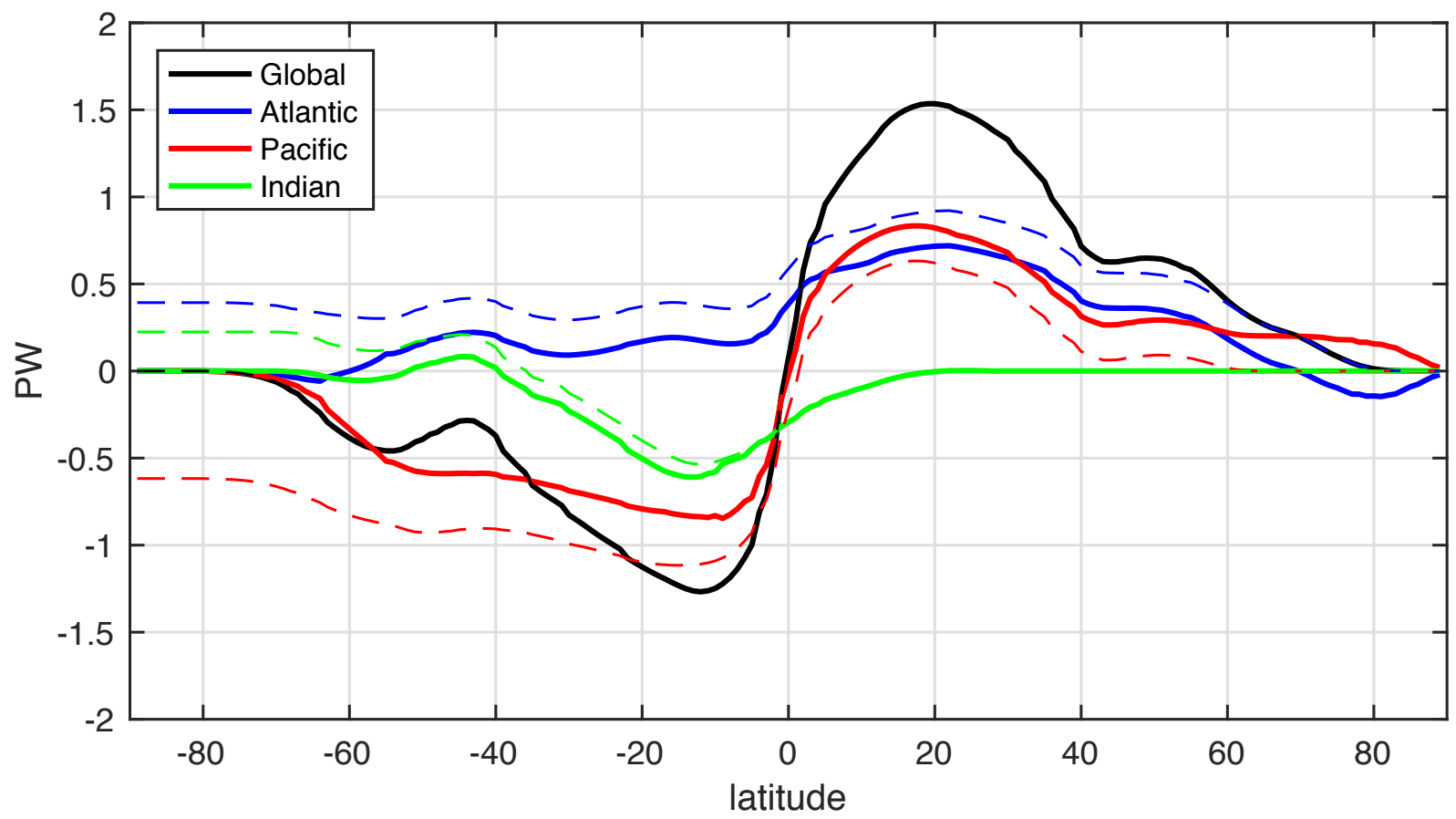

Figure S6: Meridional effOHT (solid curves; in PW) over the Globe (black), Atlantic (blue), Pacific (red), and Indian (green) sectors. Corresponding dashed curves are estimated from the same OHT convergence but based on the method of (13) (namely, infOHT). Net meridional transports integrated around the Globe are the same in both cases (black curve). See SPM section 4.1 for computational details. 

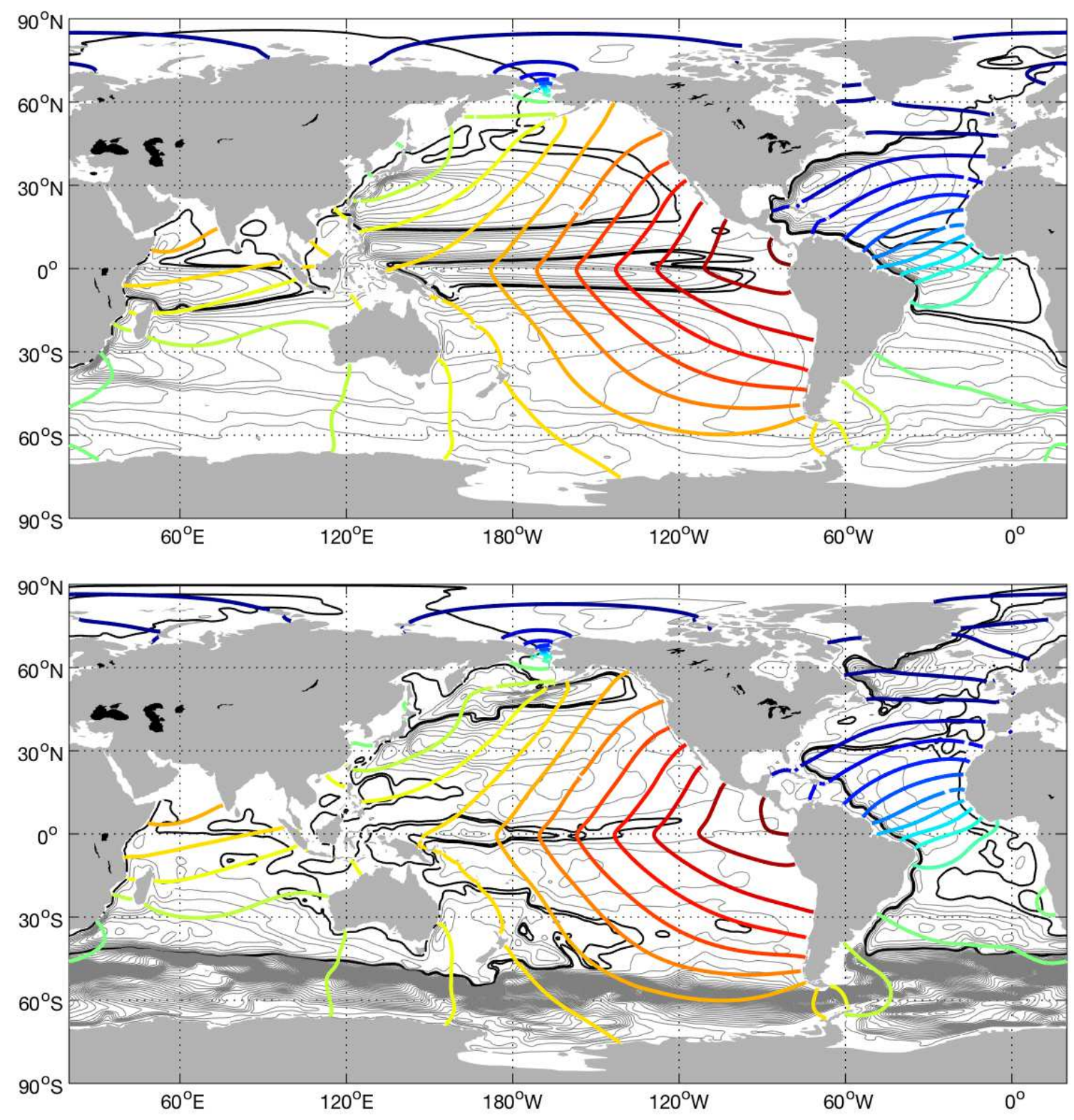

Figure S7: Same as Fig. 3 but after resetting the Energy Reference Level (ERL) to approximately the global mean ocean temperature $\left(3.5^{\circ} \mathrm{C}\right.$; top) and the global mean sea surface temperature $\left(18.5^{\circ} \mathrm{C}\right.$; bottom). See SPM section 4.2 for detail. 


\section{References}

1. G. Forget, et al., Geoscientific Model Development 8, 3071 (2015).

2. G. Forget, et al. (2016).

3. J. H. Davies, Geochemistry, Geophysics, Geosystems 14, 4608 (2013).

4. M. Balmaseda, et al., Journal of Operational Oceanography 8, s80 (2015).

5. G. Forget, R. Ponte, Progress in Oceanography pp. 173-195 (2015).

6. G. Forget, D. Ferreira, X. Liang, Ocean Science 11, 839 (2015).

7. G. Forget, ECCO version 4: an ocean state estimation framework based on the MITgcm and its adjoint. (2018).

8. K. Bryan, Journal of Geophysical Research 67, 3403 (1962).

9. B. A. Warren, Journal of Geophysical Research: Oceans 104, 7915 (1999).

10. G. Forget (2016).

11. G. Forget (2016).

12. I. G. Watterson, Journal of Atmospheric and Oceanic Technology 18, 691 (2001).

13. K. E. Trenberth, J. T. Fasullo, Geophysical Research Letters 44, 1919 (2017). 2016 GL072475.

14. M. Valdivieso, K. Haines, H. Zuo, D. Lea, Journal of Geophysical Research: Oceans 119, 394 (2014).

15. L. D. Talley, Journal of Physical Oceanography 33, 530 (2003). 
16. A. M. Macdonald, M. O. Baringer, Ocean Circulation and ClimateA 21st Century Perspective, J. G. Gerold Siedler, Stephen M. Griffies, J. A. Church, eds. (Academic Press, 2013), vol. 103 of International Geophysics, pp. $759-785$.

17. H. U. Sverdrup, Handbuch der Physik 48, 608 (1957). 